Polymer Journal, Vol. 39, No. 12, pp. 1365-1372 (2007)

(C) 2007 The Society of Polymer Science, Japan

\title{
Compatibility of Polyurethane/(vinyl ester resin)(ethyl acrylate) Interpenetrating Polymer Network
}

\author{
Chuanli QIN, ${ }^{1, \dagger}$ Zheng JIN, ${ }^{1}$ Xuduo BAI, ${ }^{1}$ Haijian JIANG, ${ }^{1}$ and Weimin $\mathrm{CAI}^{2,3}$ \\ ${ }^{1}$ Key Laboratory of Functional Polymer Materials, School of Chemistry and Chemical Engineering, \\ Heilongjiang University, Harbin 150080, People's Republic of China \\ ${ }^{2}$ Department of Environmental Science and Engineering, Shanghai Jiaotong University, \\ Shanghai 200030, People's Republic of China \\ ${ }^{3}$ Department of Environmental Science and Engineering, Harbin Institute of Technology, \\ Harbin 150001, People's Republic of China
}

(Received December 6, 2006; Accepted September 12, 2007; Published October 31, 2007)

\begin{abstract}
A novel polyurethane/poly(vinyl ester resin) interpenetrating polymer Network(PU/VER IPN), was synthesized at room temperature by polymerizing ethyl acrylate(EA) instead of the conventionally styrene(St) as a comonomer. The respective compatibilities of PU/VER(EA)IPN and PU/VER(St)IPN were studied by ATR-FT/IR, TEM, AFM, DSC and DMA. The results showed that, compared with PU/VER(St)IPN, the degree of carbonyl hydrogen bonding in PU/VER(EA)IPN increased because EA substituted for St, resulting in better compatibility of PU/ VER(EA)IPN. PU/VER(EA)IPN showed a nanometer scale phase range and a dual continuous microstructure, while the phase range of PU/VER(St)IPN was $200-500 \mathrm{~nm}$. Furthermore, DSC showed that PU/VER(EA)IPN had one obvious $T_{g}$, while PU/VER(St)IPN had two $T_{g} s$. The compatibility of PU/VER(EA)IPN was further detected quantitatively. In addition, the 60:40 PU/VER(EA)IPN had a predominant damping peak and a shoulder damping peak which were closer than the two corresponding peaks of PU/VER(St)IPN. PU/VER(EA)IPN had higher tan $\delta, E^{\prime \prime}, E^{\prime}, T A$ and $L A$ for a broad range of temperature, all indicating better compatibility and damping properties. Their mechanical properties were also studied preliminarily. [doi:10.1295/polymj.PJ2006186]

KEY WORDS Interpenetrating Polymer Networks / Polyurethane / Vinyl Ester Resin / Compatibility /
\end{abstract}

Interpenetrating polymer network(IPN) materials have potential utilities as functional materials in many applied fields. ${ }^{1,2}$ Many factors influence the IPN's properties, such as its damping and mechanical properties, and the compatibility of the network is among the more obvious and important factor. ${ }^{3}$ Although one can constrain the phase separation, change the compatibility and improve the materials' properties by making use of permanent interpenetration and entanglement in IPN, not all of the materials' compatibility aspects can be effectively improved by this technology because the compatibility of IPN is determined to a great extent by the solubility parameter difference $(\Delta \delta)$ between the components.

Since the 1980s, scientists have studied in particular the microstructure, compatibility and mechanical properties of polyurethane/(unsaturated polyester)IPN(PU/UPR IPN). ${ }^{4,5}$ Polyurethane/(vinyl ester resin)IPN (PU/VER IPN), which derived from PU/ UPR IPN, has the excellent performance of epoxy resins (EP) besides the excellent low temperature performance of PU and the radical polymerization mechanism of UPR. As a result, PU/VER IPN has attracted many researchers in recent years. ${ }^{6-8}$ In order to im- prove the compatibility of PU/VER IPN, Wang synthesized graft IPN with various types and lengths of side chains and dual IPN by using a macromonomer technique, ${ }^{9}$ while Dou introduced ionic bond to PU/ graft VER IPN with regular side chain. ${ }^{10}$ The compatibility of general PU/VER(styrene)IPN (Styrene being a comonomer) is bad due to the fact that PU and polystyrene (PSt) are incompatible in thermodynamic terms, as pointed out by $\mathrm{Kim}^{11}$ in 1975 . Researchers have tried to improve the compatibility of PU/VER IPN by changing the comonomer. Wang introduced methyl methacrylate (MMA) as a comonomer and prepared PU/VER(MMA)IPN whose compatibility, interpenetration degree and mechanical properties are better than those of PU/VER(St)IPN. ${ }^{12}$ Because the $\Delta \delta$ of polyethyl acrylate (PEA) and PU is smaller than that of PMMA and PU, ${ }^{13}$ it is thought that the compatibility and molecular composite degree of PU/ VER(EA)IPN may be excellent when adding EA with flexible pendant groups and good compatibility with PU as comonomer. Furthermore, the IPN material has the synthetical properties of PU, EP and acrylic esters and they are expected to become a novel kind of IPN damping materials with promising prospect.

${ }^{\dagger}$ To whom correspondence should be addressed (Tel: +86-451-86608131, Fax: +86-451-86608131, E-mail: chuanliqin@ 163.com). 


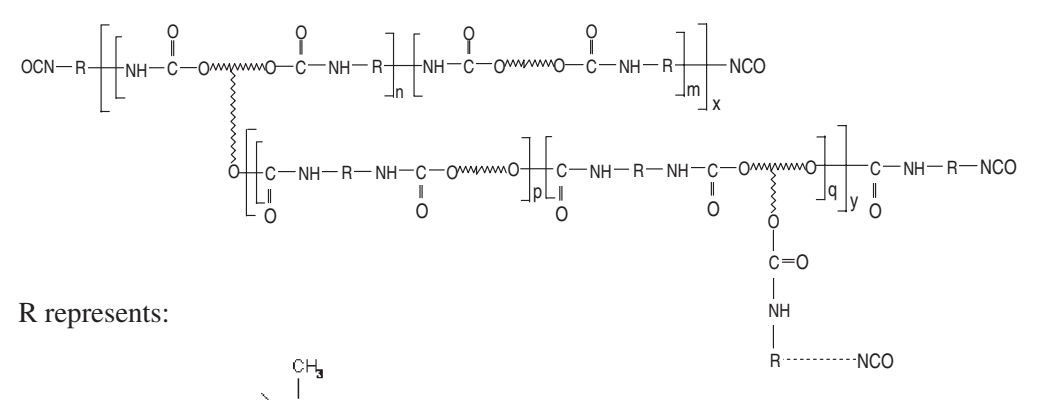

(a)

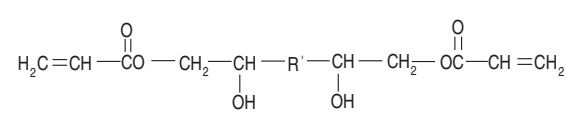

$\mathrm{R}^{\prime}$ : represents:

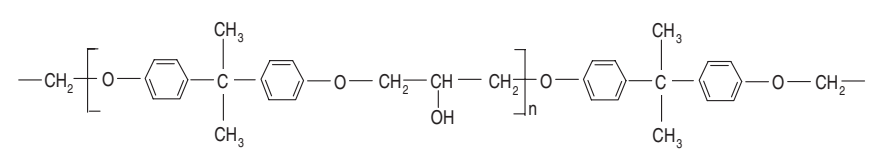

(b)

Figure 1. The molecular structures of PU prepolymer and epoxy acrylate: (a) PU prepolymer; (b) epoxy acrylate.

In this paper, PU/VER(EA)IPNs were synthesized at room temperature by polymerizing EA instead of conventional St as a comonomer with simultaneous interpenetration. The compatibility of PU/VER(EA)IPN and PU/VER(St)IPN were studied by Attenuated total reflectance Fourier transform infrared spectroscopy (ATR-FT/IR), Transmission electron microscopy(TEM), Atomic force microscopy (AFM), Differential scanning calorimetry(DSC) and Dynamic mechanical analysis(DMA). Furthermore, the mechanical properties of PU/VER(EA)IPN were measured in order to provide reference for preparing IPN materials with excellent synthetic properties.

\section{EXPERIMENTAL}

\section{Materials}

PU prepolymer was prepared in our laboratory as described elsewhere ${ }^{14}$ and its molecular structure was believed to be as shown in Figure 1(a). Epoxy acrylate was supplied by Shanghai Synthetic Resin Plant and its molecular structure was as shown in Figure 1(b). Trimethylol propane (TMP) and stannous octoate (also called T-9) respectively were supplied by Jilin Haite Chemical reagent Company and American Air Company. Styrene (St), ethyl acrylate (EA), benzoyl peroxide (BPO), 1,4-butylene glycol $(1,4-$
BD), N,N'-dimethylaniline (DMA), ethyl acetate (EAc) were standard reagent grade and obtained from Harbin Chemical Reagent Company.

TMP was dried at $100^{\circ} \mathrm{C}$ under vacuum for $3 \mathrm{~h}$. Inhibitors were removed from St, EA. Water was removed from EAc by reduced pressure distillation.

\section{Preparation of PU/VER IPN}

The calculated amount of TMP at a molar ratio of $\mathrm{NCO} / \mathrm{OH}=2.4$ (where $\mathrm{NCO}$ and $\mathrm{OH}$ refer respectively to the isocyanate group of the PU prepolymer and the hydroxyl group of TMP) as polyurethane crosslinker was dissolved in dehydrated EAc (30 wt $\%$ based on the total mass). Then 1,4-BD at a molar ratio of $\mathrm{NCO} / \mathrm{OH}=2.4$ (where $\mathrm{NCO}$ and $\mathrm{OH}$ refer respectively to the isocyanate group of the $\mathrm{PU}$ prepolymer and the hydroxyl of 1,4-BD) as PU chain-extender, together with a set amount of vinyl ester resin (which is the term used here for a mixture of $66.7 \mathrm{wt} . \%$ epoxy acrylate and $33.3 \mathrm{wt}$ \% EA or 33.3 wt. \% St), a set amount of PU prepolymer, 0.67 wt. \% BPO and 0.34 wt. \% DMA based on VER as the redox initiator system for the VER and 0.5 wt. \% T-9 based on PU as the catalyst for the PU were added. After the mixture had been thoroughly stirred and degassed under vacuum for 5 to $10 \mathrm{~min}$, a yellow, transparent sample was prepared by curing 
it at room temperature in a mould. A series of IPNs was synthesised in this way by varying the component ratios of PU and VER in the system.

\section{Measurements}

Attenuated total reflection Fourier transform infrared (ATR-FT/IR) spectroscopy was carried out on a Nicolet NEXUS 670 spectrometer. Spectra in the range from $4000 \mathrm{~cm}^{-1}$ to $600 \mathrm{~cm}^{-1}$ were collected at room temperature.

Transmission Electron Microscopy (TEM) observations were carried out using a JEM 1200-EX apparatus. The samples were cut into ultra-thin section about $100 \mathrm{~nm}$ thickness with a freezing superthin microtome in liquid nitrogen and stained with $2 \% \mathrm{OsO}_{4}$ vapor for $48 \mathrm{~h}$.

Atomic Force Microscopy (AFM) Imaging was performed on microtomed surfaces with a Digital Instrument Nanoscope III Scanning Probe Microscope with multimode head and J-scanner. The tapping mode was used in ambient conditions. Commercial Si probes with resonance frequencies in the $300 \mathrm{kHz}$ range were chosen.

Glass transition temperature $\left(T_{g}\right)$ was detected on a PERKIN-ELMER DSC-7 Calorimeter over a temperature range from -75 to $225^{\circ} \mathrm{C}$ at a heating rate of $5^{\circ} \mathrm{C} / \mathrm{min}$.

Dynamic Mechanical Analysis (DMA) was carried out on a Metravib MAK-04 Viscoanalyser over a temperature range from -75 to $100^{\circ} \mathrm{C}$ at a heating rate of $3{ }^{\circ} \mathrm{C} / \mathrm{min}$ at $11 \mathrm{HZ}$. The samples were rectangular bars $(2.0 \mathrm{~cm} \times 1.5 \mathrm{~cm} \times 0.2 \mathrm{~cm})$.

Mechanical properties were measured on a INSTRON 4467 tensile tester with extension rate of $100 \mathrm{~mm} / \mathrm{min}$. The samples were cut into the dumbbell shape in accordance with GB1040-79.

\section{RESULTS AND DISCUSSION}

\section{ATR-FT/IR Analysis}

The ordered structures of PU in the IPN are related to hydrogen bonding and can be studied using infrared spectroscopy. In fact, three characteristic absorption regions associated with hydrogen bonding exist in the IPN spectra. These are the amino region (3100$\left.3500 \mathrm{~cm}^{-1}\right)$, the carbonyl region $\left(1600-1800 \mathrm{~cm}^{-1}\right)$ and the ether link region $\left(1000-1110 \mathrm{~cm}^{-1}\right)$. Because of the complexity of the spectral bands in the amino and ether link regions, the absorption band in the carbonyl region has usually been used in the literature to evaluate the hydrogen bonding in IPN. The IR spectra of 70:30 PU/VER(EA)IPN and 70:30 PU/VER(St)IPN in the carbonyl region were shown in Figure 2. In Figure 2, multiple absorption bands could be observed. Furthermore, in the spectrum of PU/

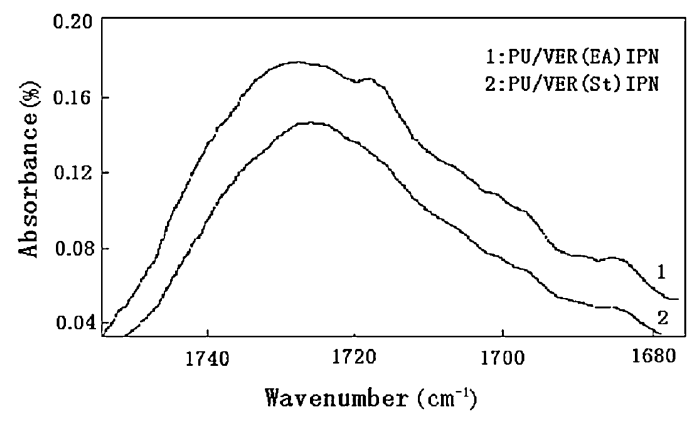

Figure 2. IR spectra of 70:30 PU/VER IPN in the carbonyl region.

Table I. The results of Least-square curve fitting in the carbonyl region

\begin{tabular}{ccccc}
\hline \multirow{3}{*}{ Samples } & \multicolumn{3}{c}{ Peak area (\%) } & $\begin{array}{c}\mathrm{X}_{\mathrm{b}, \mathrm{UT}} \\
\end{array}$ \\
\cline { 2 - 4 } & $\begin{array}{c}1731 \\
\left(\mathrm{~cm}^{-1}\right)\end{array}$ & $\begin{array}{c}1720 \\
\left(\mathrm{~cm}^{-1}\right)\end{array}$ & $\begin{array}{c}1703 \\
\left(\mathrm{~cm}^{-1}\right)\end{array}$ \\
\hline PU/VER(St)IPN & 41.66 & 19.72 & 38.62 & 59.34 \\
PU/VER(EA)IPN & 39.95 & $35.60(1718)^{*}$ & 24.45 & 60.05 \\
\hline
\end{tabular}

${ }^{*}$ The value in the bracket is measured wavenumber.

VER(EA)IPN an obvious absorption peak occurred at $1720 \mathrm{~cm}^{-1}$ which was associated with disordered hydrogen-bonding absorption.

In order to analyze the spectra quantitatively, the iteration procedure of damping least squares was used to describe the separated band contours by means of an equation, that is, a Lorentzian and Guassian combination. ${ }^{15}$ Three bands occurred at about $1731 \mathrm{~cm}^{-1}$, $1720 \mathrm{~cm}^{-1}$ and $1703 \mathrm{~cm}^{-1}$ which were respectively free carbonyl stretching absorption $\left(v_{c=o, \text { free }}^{U T}\right)$, disordered hydrogen-bonded absorption ( $\left.v_{c=o \text {,disordered }}^{U T}\right)$ and ordered hydrogen-bonded absorption $\left(v_{c=0, \text { ordered }}^{U T}\right)^{16,17}$ which was in accordance with the conclusions drawn by Harthcock ${ }^{18}$ and Strikovsky. ${ }^{19}$

The least-square, curve-fitting results were listed in Table I. The degree of hydrogen bonding $\left(X_{b, U T}\right)$ in Table I was defined as follows:

$$
X_{b, U T}=\frac{\Sigma \text { Area }(\text { bonded })}{\text { Area }\left(1735 \mathrm{~cm}^{-1}\right)+\Sigma \text { Area }(\text { bonded })}
$$

$\Sigma$ Area(bonded)—disordered and ordered hydrogenbonding absorption peak area

Area $\left(1735 \mathrm{~cm}^{-1}\right)$ - free carbonyl stretching absorption peak area near $1735 \mathrm{~cm}^{-1}$

As shown in Table I, the amount of ordered hydrogen-bonded urethane carbonyl of PU/VER(EA)IPN $\left(1703 \mathrm{~cm}^{-1}\right)$ decreased compared to that of PU/ VER(St)IPN, while the amount of disordered hydrogen-bonded urethane carbonyl $\left(1718 \mathrm{~cm}^{-1}\right)$ increased dramatically. However, as a whole, the degree of carbonyl hydrogen bonding was improved. It is suggest- 

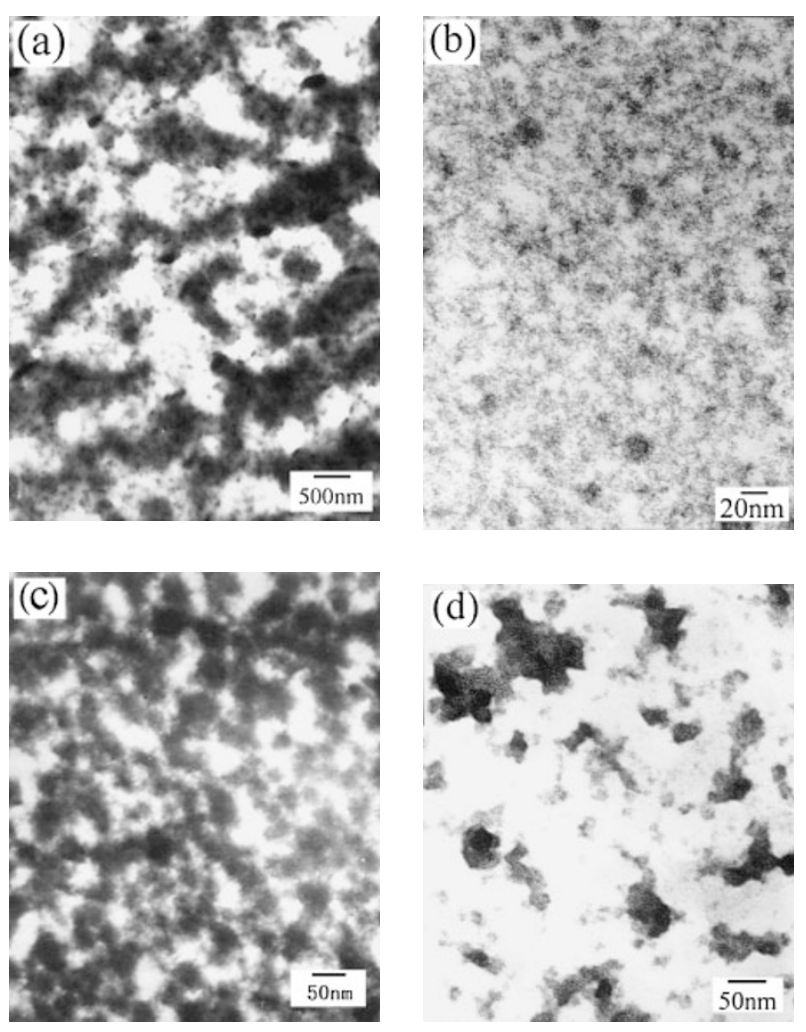

Figure 3. TEM images of PU/VER(EA)IPN and PU/VER(St)IPN: (a) 60:40 PU/VER(St)IPN; (b) 60:40 PU/VER(EA)IPN; (c) 70:30 PU/VER(EA)IPN; (d) 50:50 PU/VER(EA)IPN.

ed that this may be due to the introduced EA monomer inhibiting the formation of some ordered carbonyl hydrogen bonds between the polyurethane linkages (which was be further verified by DSC measurements shown below) which was unfavorable for the improvement of compatibility and leading to more disordered hydrogen bonds forming between the amino moiety of PU and the carbonyl of the PEA segments which was favorable for the improvement of compatibility, and therefore resulting in the increase of $X_{b, U T}$ and the compatibility improvement of PU/VER(EA)IPN. The results described here thereby affirmed the strategy presented in the present paper.

\section{Microstructure Observations}

TEM images of PU/VER(EA)IPN and PU/VER(St)IPN were displayed in Figure 3. The PU phase was shown stained by $\mathrm{OsO}_{4}$ while the VER phase was shown in white. For the 60:40 PU/VER(St)IPN (Figure 3(a)), the VER phase was dispersed in the continuous PU phase with domain sizes in the range of 200-500 nm, thereby indicating that the compatibility of PU/VER(St)IPN was poor. On the other hand, PU/VER(EA)IPN with the same component ratio (Figure 3(b)) showed the morphology of dual-phase continuity, containing some fine phase domains remaining in the nanometre range (less than $20 \mathrm{~nm}$ ).
70:30 PU/VER(EA)IPN (Figure 3(c)) and 50:50 PU/VER(EA)IPN (Figure 3(d)) showed domain sizes smaller than $100 \mathrm{~nm}$. Compared to 70:30 PU/VER(EA)IPN, the phases of 50:50 PU/VER IPN were reversed: the VER phase became the more continuous phase because of the change in component ratios, and the domain sizes of the dispersed PU phase were bigger and its continuous degree was lower, which indicated that the compatibility of the 50:50 PU/VER(EA)IPN was inferior to that of the 70:30 PU/VER(EA)IPN. According to the TEM images, it was clear that the interpenetration effect and the compatibility of PU/VER(EA)IPN were generally superior than those of PU/VER(St)IPN, the introduction of EA as a comonomer instead of St improved the compatibility between the networks of common PU/VER(St)IPN, which verified our design. Furthermore, it was mentioned that irregular phase sizes of IPN materials were due to the abundant interpenetration structure which prevented the domains from contracting to least surface area in the present of interior tension.

AFM in tapping mode can also be used to observe the microstructure and compatibility of materials using the principle that phase lag is directly related to the elastic modulus of the materials. ${ }^{20}$ The modulus difference between PU and VER is large enough to provide good contrast in AFM phase images. AFM images of the 70:30 and 50:50 PU/VER(EA)IPNs were shown in Figure 4. The bright phase was the VER phase with higher modulus and the darker phase was the PU phase with lower modulus. For 70:30 IPN, although the VER phase was dispersed in a continuous PU phase, obvious gradient distribution emerged in the interface with transition color indicating much interpenetration and good compatibility between the two phases. Furthermore, the dispersed VER phase was also linked through fine structure, however it was less continuous than the PU phase. For the 50:50 IPN, larger VER phase sizes and sharper phase boundaries emerged and the VER phase became continuous. The dispersed PU phase was also linked, but the continuous degree of dispersed phase was lower than that of the 70:30 IPN, and obvious gradient contribution didn't emerge. The results of AFM analysis indicated that the phase range of the dual-phase continuous PU/VER(EA)IPN obtained was in the nanometer scale and the compatibility of the 70:30 IPN was superior than that of the 50:50 IPN, those results being in agreement with those of TEM analysis and following DSC analysis.

\section{DSC Analysis}

The DSC curves of 70:30 PU/VER IPN without comonomer, 70:30 PU/VER(St)IPN and PU/VER(EA)IPNs with various component ratios were shown 

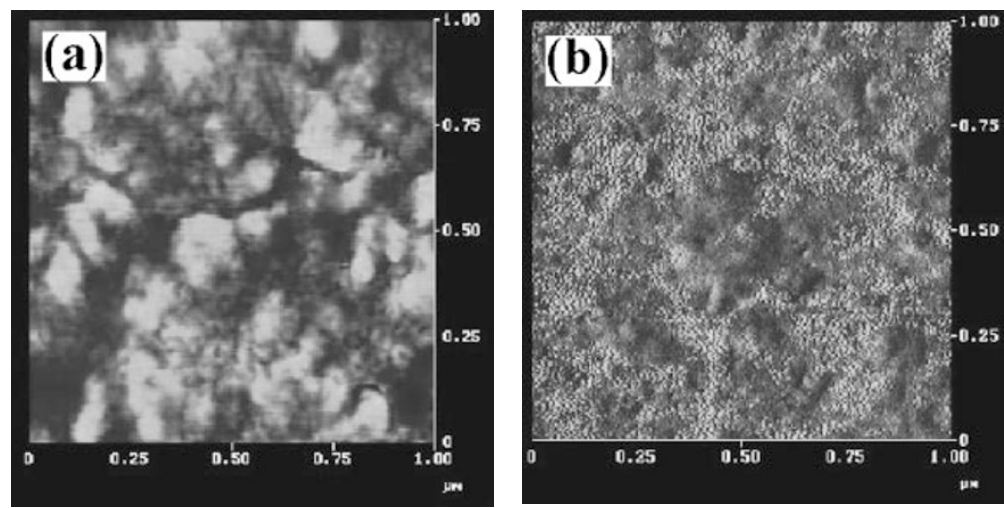

Figure 4. AFM images of PU/VER(EA)IPN with different component ratios: (a) 70:30 PU/VER(EA)IPN; (b) 50:50 PU/VER(EA)IPN.

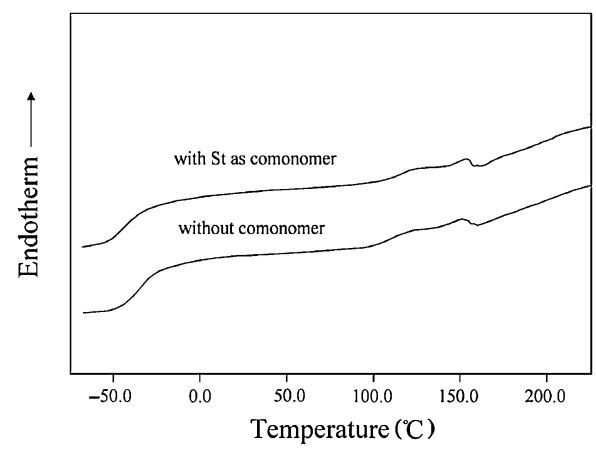

Figure 5. DSC curves of PU/VER IPN with different comonomers.

in Figures 5 and 6. As shown in Figures 5 and 6, the compatibility between the soft and hard segments of pure PU was good and the PU network exhibited a $T_{g}$ of the soft segment and an ordered dissociation peak which could be attributed to the disruption of domains composed of order hard segments ${ }^{6,21}$ due to the strong hydrogen bonding existing in the system.

There were two $T_{g}$ s for PU/VER IPN without comonomer and PU/VER(St)IPN, which were respectively hard component's $T_{g}$ and soft component's $T_{g}$, and an ordered dissociation peak of the PU hard segments. Furthermore, the samples were white, nonhomogeneous and turbid which indicated that obvious phase separation occurred in these systems. On the other hand, there was one obvious $T_{g}$ for the PU/ VER(EA)IPN with the same component ratio, the ordered dissociation peak of the PU hard segments vanished, and the sample was faint yellow, homogeneous and transparent which indicated that EA as a comonomer inhibited the formation of ordered carbonyl hydrogen bonds in the IPNs and had improved compatibility compared to that of common PU/VER(St)IPN. The reason for this compatibility improvement had been explained earlier (ATR-FT/IR analysis section) and the conclusion of DSC analysis was consistent with those of TEM and AFM.

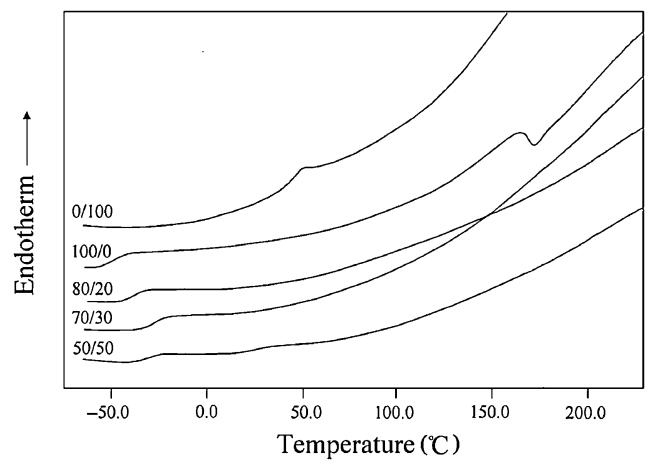

Figure 6. DSC curves of PU/VER(EA)IPN with different component ratios.

As shown in Figure 6, in the detected range of component ratios, there was either one single or two $T_{g} \mathrm{~s}$ for the PU/VER(EA)IPNs. Compared to the $T_{g} \mathrm{~s}$ of pure PU and VER, those $T_{g} \mathrm{~s}$ shifted towards one another this being due to the fact that interpenetration and entanglement in the IPN increased the compatibility of the networks and strengthened the interaction of the phases. Furthermore, there was no ordered dissociation peak for all the PU/VER(EA)IPNs indicating that the order structure of the PU hard segments in the IPNs had been destroyed and the compatibility of the networks improved.

As shown in Figure 6, the IPNs with the component ratios of 80:20 and 70:30 were compatible. For those compatible IPNs, two well-known copolymer equations can be adopted to the calculation of their theoretical $T_{g}{ }^{6}$

$$
\begin{aligned}
\frac{1}{T_{g(\text { Fox })}} & =\frac{W_{1}}{T_{g 1}}+\frac{W_{2}}{T_{g 2}} \\
T_{g(a v)} & =W_{1} T_{g 1}+W_{2} T_{g 2}
\end{aligned}
$$

where $T_{g 1}$ and $T_{g 2}$ represent the $T_{g}$ of polymer I and polymer II respectively, K. $W_{1}$ and $W_{2}$ represent their weight fractions. $T_{g(F o x)}$ and $T_{g(a v)}$ are respectively the calculated values according to the Fox equation and 


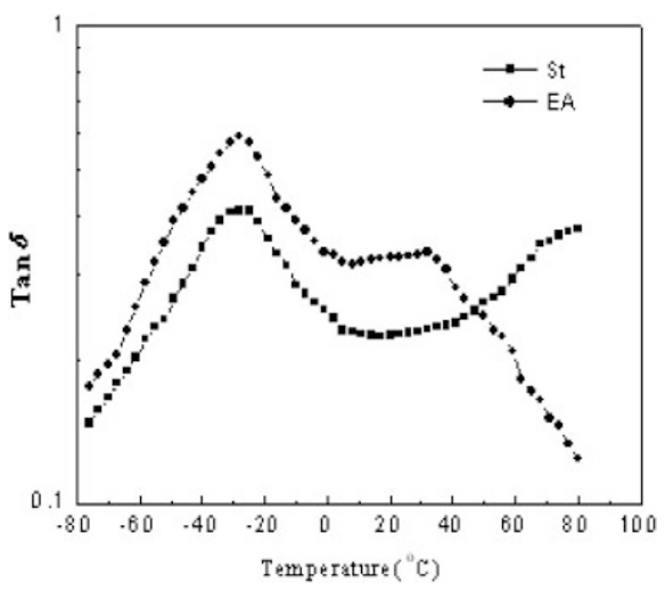

(a)

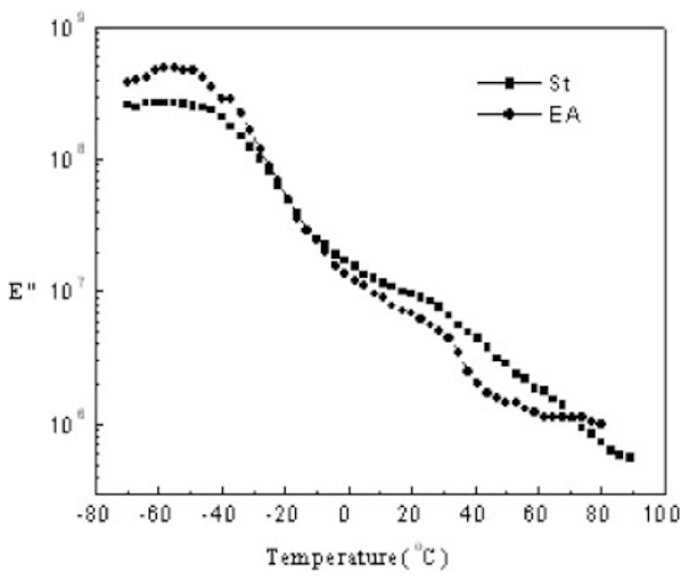

(b)

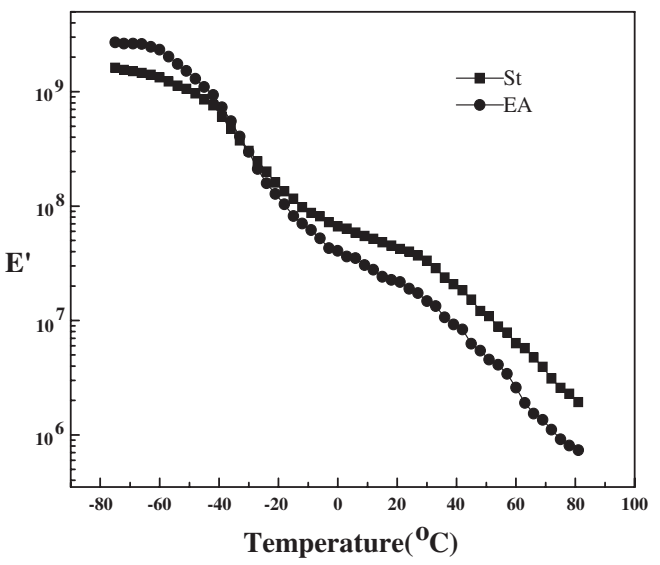

(c)

Figure 7. DMA curves of PU/VER(EA)IPN and PU/VER(St)IPN: (a) The curves between Tan $\delta$ and Temperature; (b) The curves between $E^{\prime \prime}$ and Temperature; (c) The curves between $E^{\prime}$ and Temperature.

the arithmetical mean, K. According to Figure 6, $T_{g 1}$ and $T_{g 2}$ which was the $T_{g}$ of PU and $T_{g}$ of VER(EA) respectively was $-47^{\circ} \mathrm{C}$ and $42^{\circ} \mathrm{C} . T_{g(F o x)}$ and $T_{g(a v)}$ of 80:20 IPN was $-33^{\circ} \mathrm{C}$ and $-29^{\circ} \mathrm{C}$. $T_{g(F o x)}$ and $T_{g(a v)}$ of $70: 30$ IPN was $-26^{\circ} \mathrm{C}$ and $-20^{\circ} \mathrm{C}$.

For compatible IPNs, the flexibility of the segments can be estimated by the following two equations. ${ }^{6,22}$

$$
\begin{aligned}
& \frac{T_{g(D S C)}-T_{g(a v)}}{T_{g(a v)}}=-\frac{\theta}{\theta+1} \\
& \theta=\left(F_{x} / F_{m}-1\right) X_{c}{ }^{\prime}
\end{aligned}
$$

where $X_{c}^{\prime}$ is an increase in physical crosslink density caused by interpenetration in the IPNs. $F_{x} / F_{m}$ is the ratio of the segmental mobilities for interpenetrated and non-interpenetrated network. $\theta$ is the compatible factor. $T_{g(D S C)}$ is the measured values by DSC, K.

As shown in Figure 6, $T_{g(D S C)}$ of 80:20 IPN and 70:30 IPN was $-41^{\circ} \mathrm{C}$ and $-29^{\circ} \mathrm{C}$. According to equation (3), $\theta$ of 80:20 IPN and 70:30 IPN was 0.0522 and 0.0362 . When $\theta>0, F_{x}>F_{m}$ can be estimated according to equation (4), which indicated that the mobility of the segments in IPNs was larger than that in non-interpenetrating polymer network. Because of the mobility improvement of segments, $T_{g(D S C)}$ was lower than $T_{g(F o x)}$ and $T_{g(a v)}$. The $\theta$ of 70:30 IPN was lower than that of 80:20 (a low value represents good compatibility) which indicated that the compatibility of 70:30 IPN was superior to that of 80:20 IPN.

\section{DMA Analysis}

The influence of the comonomers on the compatibility and damping properties of PU/VER IPN was shown in Figure 7. The damping properties of PU/ VER IPN with different comonomers were shown in Table II. According to the method of group contribution analysis, different construction units have different $L A$, the area under loss modulus-temperature $\left(E^{\prime \prime}-T\right)$, and the contribution of the construction units to the total damping peak area are additive, so the contribution of different comonomers to the polymer's damping peak area varies. In the paper, the $L A$ and $T A$ (the area under the curves of mechanical loss factor-temperature $(\tan \delta-T))$ were also listed in 
Table II. Damping properties of PU/VER(EA)IPN and PU/VER(St)IPN

\begin{tabular}{cccccr}
\hline comonomer & $\begin{array}{c}\text { The temperature range } \\
\text { of } \tan \delta>0.3\left({ }^{\circ} \mathrm{C}\right)\end{array}$ & $\begin{array}{c}\text { Tan } \delta \text { at the } \\
\text { maximum }\end{array}$ & $\begin{array}{c}\text { Tan } \delta \\
\text { at } 24{ }^{\circ} \mathrm{C}\end{array}$ & $L A$ & $T A$ \\
\hline St & $-43 \sim-13 \quad 62 \sim>89$ & 0.41 & 0.22 & 10.76 & 38.12 \\
EA & $-54 \sim 39$ & 0.59 & 0.33 & 15.44 & 47.83 \\
\hline
\end{tabular}

Table II in order to quantitatively compare their capacity of dissipating mechanical energy. As shown in Figure 7 and Table II, comonomers had a great influence on the compatibility and damping properties of the IPN materials. PU/VER(EA)IPN had a predominant damping peak and a shoulder damping peak which were closer than the corresponding two peaks of PU/VER(St)IPN and for a broad range of temperature, $\tan \delta, E^{\prime \prime}, E^{\prime}, L A$ and $T A$ of PU/VER(EA)IPN were also greater than those of PU/VER(St)IPN, thereby indicating that the compatibility and damping properties of PU/VER(EA)IPN were better than those of PU/VER(St)IPN.

The results may be attributed to the fact that the damping properties of IPN were mainly affected by its compatibility and molecular structure. The compatibility of PSt and PU was poor because the solubility parameter difference $(\Delta \delta)$ of PSt $\left(\delta=1.81 \times 10^{4}\right.$ $\left.\mathbf{J}^{1 / 2} \mathrm{~m}^{2 / 3}\right)$ and PU $\left(\delta=2.04 \times 10^{4} \mathbf{J}^{1 / 2} \mathrm{~m}^{2 / 3}\right)$ was large, while that of PEA $\left(\delta=1.90 \times 10^{4} \mathrm{~J}^{1 / 2} \mathrm{~m}^{2 / 3}\right)$ and PU was considerably smaller. ${ }^{13}$ As analyzed in ATR-FT/IR, PEA had polar carboxyl groups, therefore the compatibility of PU/VER(EA)IPN could be improved due to the emergence of disordered hydrogen bonds between the polar carboxyl and the amino groups of PU. The $T_{g}$ of PEA $(249.15 \mathrm{~K})$ was located between that of PU $(226.49 \mathrm{~K})$ and that of the epoxy acrylate in VER $(362.69 \mathrm{~K})$, and thus it could effectively link their $T_{g} s$. Consequently, the compatibility of PU/VER(EA)IPN was superior to that of PU/ $\operatorname{VER}(\mathrm{St}) I P N$. On the other hand, the results may also be attributed to the effects of different molecular structures on the damping properties of IPN. The PU/VER(EA)IPN had ethyl ester groups with relatively lower potential barrier and more mobility compared to the rigid phenyl groups of PU/VER(St)IPN, and thus the ethyl ester groups had greater friction force during segment movement, contributing to better damping properties.

\section{Mechanical Property Analysis}

The Stress-strain curves and the mechanical data of PU/VER(EA)IPN materials with different component ratios were shown in Figure 8 and Table III. As shown in Figure 8 and Table III, the tensile strength increased and the elongation at break decreased with the increasing VER proportion, which showed that the material changed from elastic deformation to brit-
Table III. Mechanical properties of PU/VER(EA)IPNs with different component ratios

\begin{tabular}{cccc}
\hline $\begin{array}{c}\text { PU/VER } \\
\text { (wt.) }\end{array}$ & $\begin{array}{c}\text { Tensile strength } \\
\text { (MPa) }\end{array}$ & $\begin{array}{c}\text { Elongation } \\
\text { at break }(\%)\end{array}$ & $\begin{array}{c}\text { Elastic module } \\
\text { (MPa) }\end{array}$ \\
\hline 90:10 & 1.90 & 72.40 & 13.38 \\
80:20 & 2.29 & 66.20 & 14.31 \\
$70: 30$ & 4.35 & 44.71 & 19.61 \\
$60: 40$ & 7.46 & 32.09 & 70.66 \\
$50: 50$ & 9.78 & 23.06 & 133.57 \\
$40: 60$ & 14.19 & 12.33 & 254.76 \\
\hline
\end{tabular}

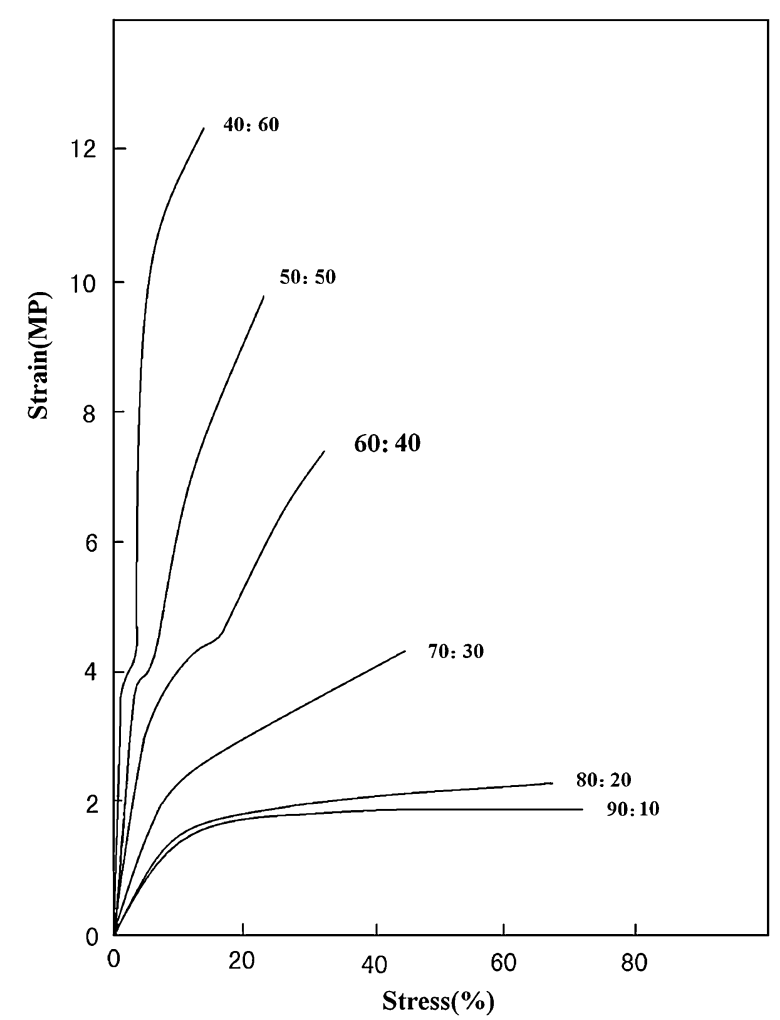

Figure 8. The Stress-strain curves of PU/VER(EA)IPN materials with different component ratios.

tle deformation. Among them the 60:40IPN, 50:50IPN and 40:60IPN showed the characteristic of rigidity and toughness. Compared to PU/VER(BMA)IPN materials, ${ }^{23}$ PU/VER(EA)IPN materials had higher tensile strength, higher Young's modulus and lower elongation at break, all showing that the effects of the VER(EA) network on the strengthening of PU were better than those of the VER(BMA) network. Presumably, this was due to the fact that the compat- 
ibility of PU/VER(EA)IPN was better than that of PU/VER(BMA)IPN according to $\Delta \delta$ (the $\delta$ of PBMA is $1.78 \times 10^{4} \mathrm{~J}^{1 / 2} \mathrm{~m}^{2 / 3}$ ) and the degree of entanglement and interpenetration was greater, thereby resulting in superior effects of VER(EA) network on the strengthening of PU.

\section{CONCLUSIONS}

PU/VER(St)IPN and PU/VER(EA)IPN were synthesized at room temperature by polymerizing respectively St and EA as comonomers with simultaneous interpenetration. The compatibilities of the two systems were studied in detail. The results showed that the PU/ VER(EA)IPN exhibited improved compatibility compared to that of conventional PU/VER(St)IPN. The introduced EA monomer inhibited the formation of ordered carbonyl hydrogen bonds, triggered the formation of disordered carbonyl hydrogen bonds, and as a whole resulted in the improvement of the degree of carbonyl bonding and better compatibility. The PU/ VER(EA)IPN showed finer morphology of the dualphase continuity with nanometer scale domains (less than $20 \mathrm{~nm}$ ) compared to that of PU/VER(St)IPN which had domain sizes in the range of $200-500 \mathrm{~nm}$. While there were two $T_{g} s$ for 70:30 PU/VER(St)IPN, there was one obvious $T_{g}$ for the 80:20 and 70:30 PU/ VER(EA)IPNs, and 70:30 PU/VER(EA)IPN also had a lower $\theta$ value, indicating better compatibility. For the broad range of temperature, PU/VER(EA)IPN had greater $\tan \delta, E^{\prime \prime}, E^{\prime}, L A$ and $T A$ than PU/VER(St)IPN and the compatibility and damping properties of PU/ VER(EA)IPN were superior to those of PU/VER(St)IPN. The synthetical mechanical properties of the IPN having components with lower $\Delta \delta$ were generally better.

We gratefully acknowledge the Nature Science Foundation Committee of Heilongjiang Province (Grant no. E2004-23), the Harbin Science Research Foundation Committee (Grant no. 2004AFXXJ046) the Youth Foundation of Heilongjiang University (Grant. NoQL200422) for financial support for this project.

\section{REFERENCES}

1. Y. Nishio, A. Yamada, K. Ezaki, Y. Miyashita, H. Furukawa, and K. Horie, Polymer, 45, 7129 (2004).

2. L. Liu and H. Sheardown, Biomaterials, 26, 233 (2005).

3. K. H. Hsieh, J. L. Han, C. T. Yu, and S. C. Fu, Polymer, 42, 2491 (2001).

4. K. H. Hsieh, J. S. Tsai, and K. W. Chang, J. Mater. Sci., 26, 5877 (1991).

5. D. Y. Tang, Z. Jin, and W. M. Cai, Mater. Chem. Phys., 82, 73 (2003).

6. N. P. Chen, Y. L. Chen, D. N. Wang, C. P. Hu, and S. K. Ying, J. Appl. Polym. Sci., 46, 2075 (1992).

7. Y. J. Wan, Y. Gu, M. L. Xie, Y. Q. Qu, X. P. Liu, W. P. Lu, and J. H. Wang, J. Funct. Polym., 13, 81 (2000).

8. C. H. Chen, W. J. Chen, M. H. Chen, and Y. M. Li, Polymer, 41, 7961 (2000).

9. G. Y. Wang, The dissertation, East China University of Science and Technology, 1999.

10. D. Y. Dou, G. Y. Wang, and C. P. Hu, Acta Chim. Sinica, 59, 1476 (2001).

11. S. C. Kim, D. Klempner, H. L. Frisch, K. C. Frisch, and H. Ghiradella, Polym. Eng. Sci., 15, 339 (1975).

12. G. Y. Wang and C. P. Hu, Acta Chim. Sinica, 59, 2012 (2001).

13. J. B. Yan and Y. K. Zhang, in "Polymer Handbook of Physical Chemistry," Petrochemistry Press, China, 1995.

14. C. L. Qin, J. S. Zhang, D. Y. Tang, and W. M. Cai, J. Nat. Sci. Heilongjiang Univ., 20, 96 (2003).

15. N. Luo, D. N. Wang, and S. K. Ying, Polymer, 37, 3577 (1996).

16. P. C. Painter, B. Veytsman, and M. M. Coleman, J. Polym. Sci., Part A: Polym. Chem., 32, 1189 (1994).

17. M. M. Coleman, K. H. Lee, and D. J. Skrovanek, Macromolecules, 19, 2149 (1986).

18. M. A. Harthcock, Polymer, 30, 1234 (1989).

19. A. G. Strikovsky and V. V. Zharkov, Polymer, 34, 3397 (1993).

20. H. Y. Chen, Y. W. Cheung, A. Hilter, and E. Baer, Polymer, 42, 7819 (2001).

21. R. W. Seymour and S. L. Cooper, Macromolecules, 6, 48 (1973).

22. A. Vilas and K. Suresh, Eur. Polym. J., 34, 1447 (1998).

23. C. L. Qin, D. Y. Tang, J. Cai, J. S. Zhang, W. M. Cai, and X. D. Sun, J. Mater. Sci. Technol., 19, 56 (2003). 